\title{
LncRNA UCA1 Promotes Mitochondrial Function of Bladder Cancer via the MiR- 195/ARL2 Signaling Pathway
}

\author{
Hui-Jin Lia Xiao-Min Sun ${ }^{a} \quad$ Zheng-Kun Lia Qian-Wen Yin ${ }^{a} \quad$ Huan Pang ${ }^{a}$ \\ Jing-Jing Pan ${ }^{\mathrm{a}} \quad \mathrm{Xu} \mathrm{Li}^{\mathrm{b}} \quad$ Wei Chen ${ }^{\mathrm{a}}$ \\ ${ }^{a}$ Clinical Laboratory, The First Affiliated Hospital, School of Medicine, Xi'an Jiaotong University, Shaanxi, \\ ${ }^{b}$ Center for Translational Medicine, The First Affiliated Hospital, School of Medicine, Xi'an Jiaotong \\ University, Shaanxi, China
}

\section{Key Words}

LncRNA UCA1 • MiR-195 • ARL2 • Mitochondria • Bladder cancer

\begin{abstract}
Background/Aims: This study aims to identify whether Urothelial Cancer Associated 1 (UCA1) regulates mitochondrial metabolic reprogramming in bladder cancer, and to explore how UCA1 participates in mitochondrial metabolism by the UCA1/miR-195/ARL2 signaling pathway; these findings may be aid in the development of tumor diagnostic and therapeutic strategies. Methods: Bladder tissues were obtained from patients. Stable cell lines were constructed, with ectopic expression of UCA1 in UMUC2 cells and knockdown of UCA1 in 5637 cells. The expression levels of UCA1, miR-195, and ARL2 were detected by real-time $P C R$, western blotting, and immunohistochemistry. Cell viability was detected by Cell Counting Kit-8 (CCK8) assay; mitochondrial DNA copy numbers were tested by realtime PCR; ATP level was evaluated by ATP assay kit; mitochondrial membrane potential was analyzed by $5,5^{\prime}, 6,6^{\prime}$-tetrachloro-1, $1^{\prime}, 3,3^{\prime}$ - tetraethylbenzimidazolylcarbocyanine iodide (JC1) fluorescent probe. miRNAs between UCA1 and ARL2 were predicted by TargetScan and RNAHybrid, and then determined by real-time PCR. Dual-luciferase activity assay and RNA immunoprecipitation (RIP) assay were used to verify the relationship between UCA1 and miR195. The expression level of ARL2 was silenced by small interfering RNA(siRNA). For in vivo experiments, UCA1-silencing 5637 cells were subcutaneously injected into BALB/C nude mice to evaluate the effects of UCA1 on tumor progression by the regulation of miR-195 and ARL2. Results: We demonstrate here that UCA1 enhances mitochondrial function in bladder cancer cells. UCA1 contributes to ARL2-induced mitochondrial activity, which plays an important role in mitochondrial function. UCA1, as a competing endogenous RNA (ceRNA), regulates mitochondrial function through upregulating ARL2. In this way, it inhibited the miR-195 signaling pathway to enhance mitochondrial function in bladder cancer. Additionally, ARL2 is a direct target of miR-195 and can be repressed by either miR-195 overexpression or UCA1 inhibition. Knockdown of ARL2 was analogous to the inhibition of UCA1 and the upregulation of miR-195. Animal experiments further indicated that UCA1 promoted bladder tumor
\end{abstract}

Wei Chen

KARGER
Clinical Laboratory, The First Affiliated Hospital,

School of Medicine, Xi'an Jiaotong University, Shaanxi 710061, (China)

Tel. 86-029-85324052, E-Mail chenwei6311@163.com 
growth by regulating miR-195 /ARL2. Conclusion: These data suggest that UCA1 enhanced mitochondrial function and cell viability through the UCA1/miR-195/ARL2 axis in vitro and in vivo. The elucidation of this signaling network provides a more adequate theoretical basis for understanding the molecular pathology of bladder cancer, and also UCA1 as a potential diagnosis and treatment target for bladder cancer.

(C) 2017 The Author(s)

Published by S. Karger AG, Basel

\section{Introduction}

Bladder cancer (BC) is one of the most common malignant cancers of the urinary system in China, and its incidence and mortality rates have increased in recent years [1]. Despite advances in the prevention and treatment of $\mathrm{BC}$, the molecular mechanism and effective diagnostic biomarkers for this cancer have yet to be identified.

Deregulation of cellular energetics is a hallmark of malignant cancer cells, mainly exhibited as enhanced absorption and utilization of glucose and glutamine [2,3]. Otto Warburg first proposed that cancer cells prefer to glycolysis mainly stemmed from dysfunctional mitochondria [4]. In contrast, changes in mitochondrial structure and function in tumor cells lead to increased absorption and utilization of glutamine. This means they are able to meet the bioenergetic and biosynthetic demands of tumor cell growth, and this is known as tumor mitochondrial metabolic reprogramming. Increasing evidence has shown that some cancer subtypes may rely more on their intact mitochondrial respiration, which plays a pivotal role in tumor progression, and the molecular mechanisms underlying metabolic reprogramming of cancer cells has become an important topic in cancer research.

Long noncoding RNAs (lncRNAs) are defined as non-protein-coding RNA molecules larger than 200 nucleotides. IncRNAs play important regulatory roles in epigenetic modification, transcription, and post-transcriptional processing [5-8]. More investigations have revealed that IncRNAs exercise their roles in the processes of tumor cell growth, differentiation, apoptosis, and metastasis. However, there are few studies showing lncRNAs participating in cancer mitochondrial metabolic reprogramming.

Studies from our, and other, laboratories have demonstrated that UCA1 is highly expressed in bladder carcinoma. UCA1 promotes BC progression through the PI3K/Akt/ CREB pathway [9], enhances BC migration and invasion by the miR-145/FSCN1/Zeb1/Zeb2 network [10], and is involved in regulation of the Warburg effect through the mTOR/STAT3/ miR-143 /HK2 signaling pathway [11]; these findings strongly demonstrate that UCA1 acts as an oncogene in $\mathrm{BC}$ development. The results showed that UCA1 participates in regulation of the Warburg effect in BC. However, it remains unclear whether UCA1 participates in mitochondrial metabolic reprogramming in $\mathrm{BC}$.

ADP-ribosylation factor-like 2 (ARL2) is a member of the ARF family, which is highly conserved in eukaryotes [12]. ARL2 plays essential roles in both cytosol and mitochondria, and ARL2 in cytosol may be recruit to mitochondria [13]. ARL2 is present in the inner membrane space of mitochondria, and is an activator of ATP/ADP transporters [14]. It has been reported that ARL2 expression is directly regulated by miR-195 in human embryonic stem cell-derived neural progenitor cells [15]. However, the expression levels of ARL2 in BCs and the molecular mechanisms involved have not yet been elucidated.

In this study, we aimed to reveal an underlying molecular mechanism of IncRNA UCA1 in mitochondrial metabolic reprogramming in BC. We found that over-expression of UCA1 significantly enhanced mitochondrial function. Furthermore, we investigated the signaling pathway participating in regulation of mitochondrial activity. We predicted that UCA1 may harbor a conserved miR-195-5p cognate recognition site through the RNAHybrid website, and we formed a hypothesis that UCA1 acts as a ceRNA for miR-195-5p. Then, we sought different target genes for miR-195 which were associated with mitochondria, and found that ARL2 contained four conserved miR-195 cognate sites and was a potential target of miR195. Collectively, it is concluded that UCA1 could promote BC cell proliferation by elevating mitochondrial function via the UCA1-miR-195-ARL2 signaling pathway. 


\section{Cellular Physiology Cell Physiol Biochem 2017;43:2548-2561 \begin{tabular}{l|l|l} 
and Biochemistry & Dublished online: November 02, 2017 & $\begin{array}{l}\text { C } 2017 \text { The Author(s). Published by S. Karger AG, Basel } \\
\text { www.karger.com/cpb }\end{array}$
\end{tabular}}

\section{Materials and Methods}

\section{Patient specimens}

BC tissues $(n=22)$, adjacent tissues $(n=10)$ and normal bladder tissue $(n=6)$ were obtained from the First Affiliated Hospital of Xi'an Jiaotong University in China between 2006 and 2011. All human samples were used in accordance with the policies of the institutional review board of the First Affiliated Hospital of Xi'an Jiaotong University. None of patients received chemotherapy or radiotherapy before surgical resection. All tissues were obtained either from transurethral resection or cystectomy. The clinicopathologic characteristics of the informative cases are shown in Table 1.

\section{Cell culture}

Two human bladder cancer cell lines (5637, UMUC2) were purchased from the American Type Culture Collection (ATCC, Manassas, VA, USA). Stable cell lines with ectopic expression of UCA1 in UMUC2 cells, named as pcDNA-M and pcDNA-U (constructed with pcDNA3.1(+)/Mock and pcDNA3.1(+)/UCA1, respectively), and cell lines with knockdown of UCA1 in 5637 cells, defined as pRNAT-N and pRNAT-U (pRNAT-U6.1/Neo-NC and pRNAT-U6.1/Neo-shUCA1, respectively) were constructed and selected in our laboratory. All cell lines were cultured in PRMI 1640 medium with L-glutamine (Gibco-BRL, Gaithersburg, MD, USA) supplemented with $10 \%$ bovine calf serum in humidified air at $37^{\circ} \mathrm{C}$ with $5 \% \mathrm{CO}_{2}$.

\section{microRNA mimics, siRNAs and plasmids transfection}

miR-195-5p mimics and miRNA negative control were purchased from RiboBio Biotech Co Ltd. (Guangzhou, China). siRNAs against ARL2 were obtained from GenePharma (Shanghai, China), sequences of siRNAs are as follows: siARL2\#1, 5'-TGCGCTGTCCACTACCCAGATGAGG-3'; siARL2\#2, 5'-TCTTCAGGATGGTTGTCTTTCCAGC-3'; siARL2\#3, 5'-TGTTCAGCTTGAATCCTCGGTGCTC-3'. Si-Ctrl was purchased from GenePharma (Shanghai, China) in parallel experiments as negative control. Cells were seeded into 6-well plates until 30\%-50\% confluence after about $24 \mathrm{~h}$ and then transfected with $50 \mathrm{nM}$ miR195-5p mimics, $5 \mu \mathrm{g}$ siRNA and corresponding negative control using the X-tremegene siRNA Transfection Reagent (Roche, Indianapolis, IN, USA) according to the protocol of the manufacturer. Cells were seeded into 6-well plates to reach $70 \%-90 \%$ confluence and co-transfected with $2 \mu \mathrm{g}$ plasmids or empty vector and $50 \mathrm{nM}$ miR-195-5p mimics or miRNA negative control using the HP X-treme Transfection Reagent (Roche, Indianapolis, IN, USA) according to the protocol of the manufacturer.

\section{Real-time PCR}

Total RNA was extracted by TRIzol reagent (Invitrogen, Carlsbad, CA, USA) or RNAFast200 (Fastagen, Shanghai, China). The RNA concentration was measured by NanoDrop2000 (Thermo Fisher ScientificInc, Massachusetts, USA) and stored at $-80^{\circ} \mathrm{C} .1-3 \mu \mathrm{g}$ RNA was reverse transcribed to cDNA using the RevertAid First Strand cDNA Synthesis Kit (Takara, Dalian, China), the miRNA reverse transcription was carried out with their primers respectively (RiboBio Biotech, Guangzhou, China). Quantitative realtime PCR was performed on the CFX96 Touch Real-Time PCR Detection System (Bio-Rad Laboratories, Hercules, CA, USA). The primer sequences for $\beta$-actin (F: 5'-TCCCTGGAGAAGAGCTACGA-3', R: 5'-AGCACTGTGTTGGCGTACAG-3'), UCA1 (F: 5'-CTCTCCATTGGGTTCACCATTC-3', R: 5'-GCGGCAGGTCTTAAGAGATGAG-3') and ARL2 (F: 5'-GAAGCAGAAAGAGCGGGA -3', R: 5'- CTGTGAAAATGCGGCTGGA-3') were synthesized from BGI Sequencing. The primers of miR-195 and U6 were purchased from RiboBio Biotech Co Ltd. The conditions for PCR reaction were as

Table 1. Clinicopathologic characteristics of bladder cancer patients

\begin{tabular}{lccccc}
\hline Number & Gender & Age (years) & Grade & TNM & Operation mode \\
\hline 1 & Male & 54 & I & TaN0M0 & Partial cystectomy \\
2 & Male & 72 & I & T1N0M0 & Radical cystectomy \\
3 & Male & 76 & I & T3aN0M0 & Radical cystectomy \\
4 & Male & 56 & I & T1N0M0 & Radical cystectomy \\
5 & Male & 48 & I & T3bN2M0 & Radical cystectomy \\
6 & Male & 62 & I & T1N0M0 & Radical cystectomy \\
7 & Male & 66 & I & T1N0M0 & Radical cystectomy \\
8 & Male & 76 & II & TaN0M0 & Partial cystectomy \\
9 & Female & 58 & II & T1bN0M0 & transurethral resection \\
10 & Female & 53 & II & T1bN0M0 & transurethral resection \\
11 & Female & 68 & II & T1N0M0 & Radical cystectomy \\
12 & Male & 68 & II & T1N0M0 & Radical cystectomy \\
13 & Female & 64 & II & T1bN0M0 & Radical cystectomy \\
14 & Male & 44 & II & T3aN0M0 & Radical cystectomy \\
15 & Male & 63 & III & T2N0M0 & Radical cystectomy \\
16 & Female & 70 & II & T1N0M0 & Radical cystectomy \\
17 & Male & 67 & III & T2N0M0 & Radical cystectomy \\
18 & Male & 58 & I & T1bN0M0 & Radical cystectomy \\
19 & Male & 75 & II & T2N0M0 & Radical cystectomy \\
20 & Male & 67 & III & T2N0M0 & Radical cystectomy \\
21 & Male & 53 & II & T2N0M0 & Radical cystectomy \\
22 & Male & 55 & III & T4N2M0 & Radical cystectomy \\
\hline
\end{tabular}




\section{Cellular Physiology Cell Physiol Biochem 2017;43:2548-2561

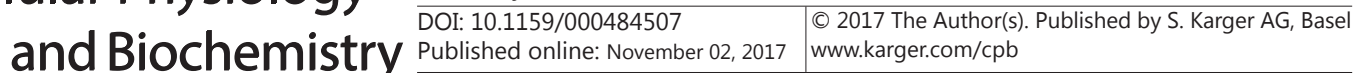

Li et al.: UCA1/MiR-195/ARL2 in Mitochondria of BC

follows: initial denaturation at $95^{\circ} \mathrm{C}$ for $10 \mathrm{~s}$, followed by 40 cycles of $95^{\circ} \mathrm{C}$ for $5 \mathrm{~s}, 57^{\circ} \mathrm{C}$ or $55^{\circ} \mathrm{C}$ for $30 \mathrm{~s}\left(57^{\circ} \mathrm{C}\right.$ for miR-195; $55^{\circ} \mathrm{C}$ for ARL2 and UCA1) and $72^{\circ} \mathrm{C}$ for $20 \mathrm{~s}$. $\beta$-actin (for UCA1 and ARL2) or U6 (for miR-195) as the internal control. The relative expression of the target gene was detected using the $2^{-\Delta \Delta \mathrm{Ct}}$ method, $\Delta \mathrm{Ct}$ $=\mathrm{Ct}$ (target gene) $-\mathrm{Ct}$ (reference), $\Delta \Delta \mathrm{Ct}=\Delta \mathrm{Ct}$ (the experimental group) $-\Delta \mathrm{Ct}$ (the control group). The data were processed by GraphPad Prism 5 statistical software.

\section{Cell viability assay}

Stable cell lines or the transfected cells at $4 \times 10^{3}$ per well were placed in triplicates in 96 -well plates and incubated for 24, 48, 72 or 96 h. Cell viability was determined by CCK-8 (7Sea, Shanghai, China), according to the manufacturer's instructions. $1.5 \mathrm{~h}$ after adding CCK-8, cell growth viability was measured by reading OD450 nm using an EnSpire Reader (PerkinElmer, USA). The images were processed by GraphPad Prism 5 statistical software.

Colony formation assay

Stable cell lines or the transfected cells at a density of $2 \times 10^{2}$ per well were seeded in the 6-well plates. After 7-10 days, cells were fixed with methanol, stained with $0.1 \%$ crystal violet. Visible colonies were scored under a microscope (Olympus Corporation, Tokyo, Japan). The images were processed by GraphPad Prism 5 statistical software.

\section{Mitochondria copy number test}

Total RNA was extracted by TRIzol reagent or RNAFast200. The RNA concentration was measured by NanoDrop 2000 and stored at $-80^{\circ} \mathrm{C}$ for further use. 1-3 $\mu$ g RNA was reverse transcribed to cDNA using the RevertAid First Strand cDNA Synthesis Kit. Mitochondrial DNA (mtDNA) was assessed by real-time quantitative PCR, using mitochondrial D-loop as target gene, normalized as 18S rRNA. The conditions for PCR were: $95^{\circ} \mathrm{C}$ for $5 \mathrm{~min}$, followed by 40 cycles of $95^{\circ} \mathrm{C}$ for $30 \mathrm{~s}, 55^{\circ} \mathrm{C}$ for $30 \mathrm{~s}$ and $72^{\circ} \mathrm{C}$ for $20 \mathrm{~s}$. The primer sequences for D-loop (F: 5'-AAGTGGCTGTGCAGACATTC-3', R: 5'-TCTGTCTTTGATTCCTGCCT-3') and 18S rRNA (F: 5'-TCTCCTACTTGGATAACTGTGG-3', R: 5'-GGCGACTACCATCGAAAGTTG-3'). mtDNA content was quantitied as the ratio of mitochondrial D-loop versus $18 \mathrm{~S}$ rRNA. The data and images were processed by GraphPad Prism 5 statistical software.

ATP assay

ATP concentrations were tested with enhanced ATP assay kit (Beyotime, Shanghai, China) according to the manusfactuer's protocol. Cells were lysed with ATP lysis buffer $(200 \mu \mathrm{L}$ of lysate per well in 6-well plates) and centrifuged at $15,000 \mathrm{~g}$ for $5 \sim 10 \mathrm{~min}$ at $4^{\circ} \mathrm{C}$. The supernatants were collected and stored on ice. Before ATP test, $100 \mu \mathrm{L}$ of ATP working solution (ATP test solution: ATP test diluention=1:5) was added to $1.5 \mathrm{~mL}$ EP tubes and incubated for $3 \sim 5 \mathrm{~min}$ at room temperature.Next the supernatant transferred to $100 \mu \mathrm{L}$ of ATP working solution. mixed quickly, and the amount of luminescence emitted was measured with a luminometer (Promega, Madison, WI, USA) immediately. The luminescence data were normalized against those sample protein amounts. The data and images were processed by GraphPad Prism 5 statistical software.

\section{Mitochondrial membrane potential (JC-1)}

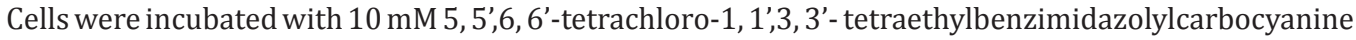
iodide (JC-1) (Beyotime, Shanghai, China) at $37^{\circ} \mathrm{C}$ for $30 \mathrm{~min}$. The fluorescence labeled cells were washed with phosphate Buffered Saline (0.01M, pH 7.4) and analyzed by an EnSpire Reader (excitation: $485 \mathrm{~nm}$; emission: $530 \mathrm{~nm}, 590 \mathrm{~nm}$ ). The ratio of fluorescence at $590 \mathrm{~nm}$ versus $530 \mathrm{~nm}$ emission was used for measuring the mitochondrial membrane potential. The data and images were processed by GraphPad Prism 5 statistical software.

The relationship of RNA expression levels

A total of 28 tissues, consisting of BC tissues $(n=22)$ and normal bladder tissue $(n=6)$ were obtained from the First Affiliated Hospital of Xi'an Jiaotong University in China between 2006 and 2011. All human samples were used in accordance with the policies of the institutional review board of the First Affiliated Hospital of Xi'an Jiaotong University. All tissues were obtained either from transurethral resection or cystectomy. Total RNA was extracted and quantitative real-time PCR was performed as previously described. 


\section{Cellular Physiology Cell Physiol Biochem 2017;43:2548-2561 \begin{tabular}{l|l} 
and Biochemistry 10.1159/000484507 & $\begin{array}{l}\text { (C) 2017 The Author(s). Published by S. Karger AG, Basel } \\
\text { www.karger.com/cpb }\end{array}$
\end{tabular}}

Li et al.: UCA1/MiR-195/ARL2 in Mitochondria of BC

The relative expression of the target gene was detected using the $\Delta \mathrm{Ct}$ value, $\Delta \mathrm{Ct}=\mathrm{Ct}$ (target gene) $-\mathrm{Ct}$ (reference). The data were processed by GraphPad Prism 5 statistical software.

\section{Bioinformatic analysis}

To examine the predicted target genes and their conserved sites that match the seed region of each miRNA, we employed the TargetScan program (http://www.targetscan.org/vert_71/). The sequences of the predicted mature miRNAs were verified by miRBase. RNAhybrid (https://bibiserv.cebitec.uni-bielefeld. de/rnahybrid/) was used to predict miRNA-binding sites in UCA1. In view of the relationship of negative correlation between the levels of UCA1 and miR-195 in bladder tissues, next we screened candidate target genes of miR-195 by bioinformatics analysis, to determine which may be participated in modulating mitochondrial function. TargetScan program revealed that GLUD1 and ARL2 were ideal targets of miR-195, mRNA and protein levels of GLUD1 and ARL2 were further detected, and the results indicated that GLUD1 expression levels were not abolished by treatment with miR-195 mimics (unpublished data). On the other hand, miR-195 expression was negatively correlated with ARL2 expression. Collectively, miR-195 and ARL2 as putative targets.

\section{Plasmid construction and Dual-luciferase assay}

Through an analysis of the complementary sequences between the miR-195-5p and UCA1, miR-195 binding sites in UCA1 have been found to contain 18 nucleotides that complement bases 411-428 of the UCA1. Thus, UCA1 fullength cDNA were subclonedinto the PMIR-REPORT ${ }^{\mathrm{TM}}$ Luciferase (pMIR-R-L) vector. The primer of pMIR-Rep-Luciferase-UCA1-miR-195 Mut (F: 5'- caacccttaagctcccaatgatatttgattggagccatgcttccatgca-3', R: 5'- tgcatggaagcatggctccaatcaaatatcattgg gagcttaagggttg-3') were synthesized from BGI Sequencing. Target fragment and mutant inserts were verified by sequencing.

To perform dual-luciferase reporter assay, Renilla luciferase plasmid serves as normalization. The cells were co-transfected in 24-well plates utilizing the X-treme GENE siRNA Transfection Reagent according to the manufacturer's instructions with $0.18 \mu \mathrm{g}$ pMIR-R-L vector and $0.02 \mu \mathrm{g}$ Renilla luciferase plasmid. Moreover, $50 \mathrm{nM}$ of miR-195-5p mimics or miRNA negative control were used for each well. Ratios of pMIRR-L/Renilla luciferase were measured using the dual-luciferase reporter assay system (Promega, Madison, WI, USA), $24 \mathrm{~h}$ after transfection. The data and images were processed by GraphPad Prism 5 statistical software.

\section{RNA immunoprecipitation (RIP)}

RIP experiments were carried out utilizing the Magna RIP RNA-binding protein immunoprecipitation kit and Ago2 antibody (Millipore, Billerica, MA, USA) following the manufacturer's specification. UMUC2 cells at about $90 \%$ confluence were scraped off and lysed in complete RNA lysis buffer (100 $\mu \mathrm{L}$ of RIP lysis buffer, added $0.5 \mu \mathrm{L}$ of protease inhibitor cocktail and $0.25 \mu \mathrm{L}$ of RNase inhibitor). $100 \mu \mathrm{L}$ of supernatant of RIP lysate was incubated with the RIP buffer ( $860 \mu \mathrm{L}$ of RIP Wash buffer, $35 \mu \mathrm{L}$ of 0.5M EDTA, $5 \mu \mathrm{L}$ of RNase inhibitor) containing magnetic beads coated with human anti-Argonaute 2 (Ago2) antibody, negative control group consisted of normal mouse IgG (Millipore, Billerica, MA, USA). After incubated at $4^{\circ} \mathrm{C}$ overnight, the co-precipitated RNA was isolated. The RNA was detected by real-time PCR. Total RNAs (input controls) and IgG were assayed simultaneously to test whether the detected signals resulted from RNAs specifically binding to Ago2. The data and images were processed by GraphPad Prism 5 statistical software and Image J sofeware.

\section{Western blotting}

Cell were lysed with RIPA lysis buffer(ComWin Biotech, Beijing, China) containing $1 \mathrm{mM}$ PMSF and $1 \%$ protease inhibitor cocktail (Roche Applied Science; Indianapolis, IN, USA). Protein concentrations were quantified by a BCA protein assay kit (ComWin Biotech, Beijing, China). Equal amount of protein was separated by SDS-PAGE, transferred to NC membranes (Pall Life Science, Port Washington, NY, USA), and blocked in 5\% nonfat milk TBS buffer for $1 \mathrm{~h}$ at room temperature. Then the membranes were incubated with anti-ARL2 antibody (1:500, Abcam; Cambridge, MA, USA), anti-SDHA, anti-HSP60, anti-PDH, antiPHB1, anti-VDAC, anti-SOD1, anti-COX IV, anti-Cytc (1:500, Cell Signaling Technology; Beverly, MA, USA), anti- $\beta$-actin (1:1000, Cell Signaling Technology; Beverly, MA, USA) primary antibodies overnight at $4^{\circ} \mathrm{C}$. Next, membranes were incubated with goat-anti-mouse or goat-anti-rabbit secondary antibody conjugated with horseradish peroxidase (HRP) (1:2000, Pierce; Rockford, IL, USA) for $1 \mathrm{~h}$ at room temperature. The 


\section{Cellular Physiology Cell Physiol Biochem 2017;43:2548-2561

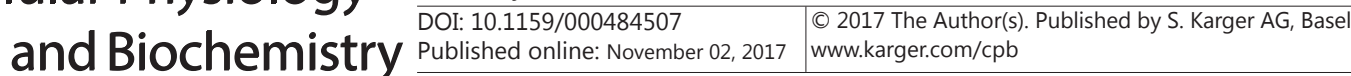

Li et al.: UCA1/MiR-195/ARL2 in Mitochondria of BC

signals were visualized by enhanced chemiluminescence detection kit (Millipore, Billerica, MA, USA) and analyzed by ChemiDoc MP System (Bio-Rad, Richmond, CA, USA). The images were processed by Image J sofeware.

\section{Immunohistochemistry (IHC)}

Immunohistochemical analysis of ARL2 was performed on BC specimens from nude mice. Samples were fixed in 10\% neutral buffered formalin, dehydrated and embedded in paraffin. The paraffin-embedded tissues were cut into five-micron-thick serial sections. Conventional dewaxing, antigenic microwave thermal retrieval, and quenching endogenous peroxidase activity were performed on sections. Primary antibodies (anti-ARL2 antibody, 1:100; Abcam, Cambridge, MA, USA) were applied to the sections at $4{ }^{\circ} \mathrm{C}$ overnight. Next, the sections were treated with secondary antibody for 30 minutes at room temperature. Polymerase auxiliary agent was then added and incubated for another 30 minutes at room temperature and stained with 3, 3'-diaminobenzidine (DAB). Finally, the slides were counterstained with $0.02 \%$ Hematoxylin and tested by microscope. The images were processed by photoshop.

\section{Animal experiments}

BALB/c athymic nude mice (female, 4-6-weeks old) were purchased from Animal center of Xi'an Jiaotong University. pRNAT-U and pRNAT-N cells $\left(2 \times 10^{7}\right.$ cells $)$ were subcutaneously injected into the flanks of mice. Tumor growth was measured weekly. Tumor volumes were examined by the following formula: $\mathrm{V}=$ length $\times$ width $^{2} / 2.4$ weeks after cells injection, the mice euthanized and the tumors were excised for analysis. The data and images were analyzed by GraphPad Prism 5 statistical the images and photpshop.

\section{Statistical analysis}

All the experiments were performed three times independently. Data are presented as the means \pm SEM, were analyzed using one-way ANOVA by the SPSS 18.0 and GraphPad Prism 5 statistical software. A value of $\mathrm{P}<0.05$ was considered to be significant.

\section{Results}

UCA1 is upregulated in BC tissues and cell lines, and associated with BC progression and cell growth

To identify whether UCA1 was differentially expressed in BC tissues, real-time PCR analysis was performed to detect the expression levels of UCA1 in BC tissues ( $n=22)$, adjacent tissues $(n=10)$, and normal bladder tissues $(n=6)$. We determined that UCA1 was upregulated in cancer tissues, and UCA1 expression in cancer tissues was significantly higher than that of adjacent tissues $(P<0.05$; Fig. $1 \mathrm{~A})$. Meanwhile, we found that UCA1 expression levels in G1 were higher than normal bladder tissues $(P<0.05$; Fig. $1 \mathrm{~B})$; and in non-muscle-invasive $\mathrm{BC}$ were higher than normal tissues $(P<0.05$; Fig. $1 C)$. These results suggest that the expression of UCA1 may be associated with pathological grade and clinical stage. Moreover, an earlier study in our laboratory demonstrated that UCA1 was more highly expressed in 5637 and BLZ-211 cell lines than in UMUC2 and BLS-211 cell lines [16]. These results revealed that UCA1 may participate in initiation, development, and prognosis of BC.

Previously, we constructed models of overexpression of UCA1 in UMUC2 cells and downregulation of UCA1 in 5637 cells. UCA1 was upregulated after pcDNA3.1/UCA1 (94.3fold increase) in UMUC2 cell lines, while UCA1 expression was significantly inhibited by pRNAT-U6.1/Neo-shUCA1 (58.7\% decrease) in 5637 cell lines [16]. To further explore the effect of UCA1 expression on BC cell growth, the CCK8 assay was performed to determine the effects of upregulation and knockdown of UCA1 expression on cellular proliferation. The results indicated that cell viability of pcDNA-U was significantly higher than that of pcDNA-M. Meanwhile, there was a significant repression in pRNAT-U compared with pRNAT-N (Fig. 1D). Significant differences were detected at different time points (72h, 96h; Fig. 1D). In line with these results, the colony-formation assay implied that colony number was significantly increased in overexpression of UCA1 in UMUC2 cell lines. Similarly, clonogenic survival reduced with the knockdown of UCA1 in 5637 cell lines (Fig. 1E). These results demonstrated that UCA1 markedly promoted cellular proliferation in BC cells. 
Fig. 1. UCA1 is up-regulated in $\mathrm{BC}$ tissues and cell lines, and a s s o ciated with BC progression and cell growth. (A) Relative expression of UCA1 expression in BC tissues $(n=10)$ and in paired adjacent tissues $(n=10)$. UCA1 expression was determined by real-time PCR and normalized to $\beta$-actin expression. (B) Relative expression of UCA1 in Grade1 (G1) $(\mathrm{n}=8)$ and normal bladder tissues (n=14). (C)
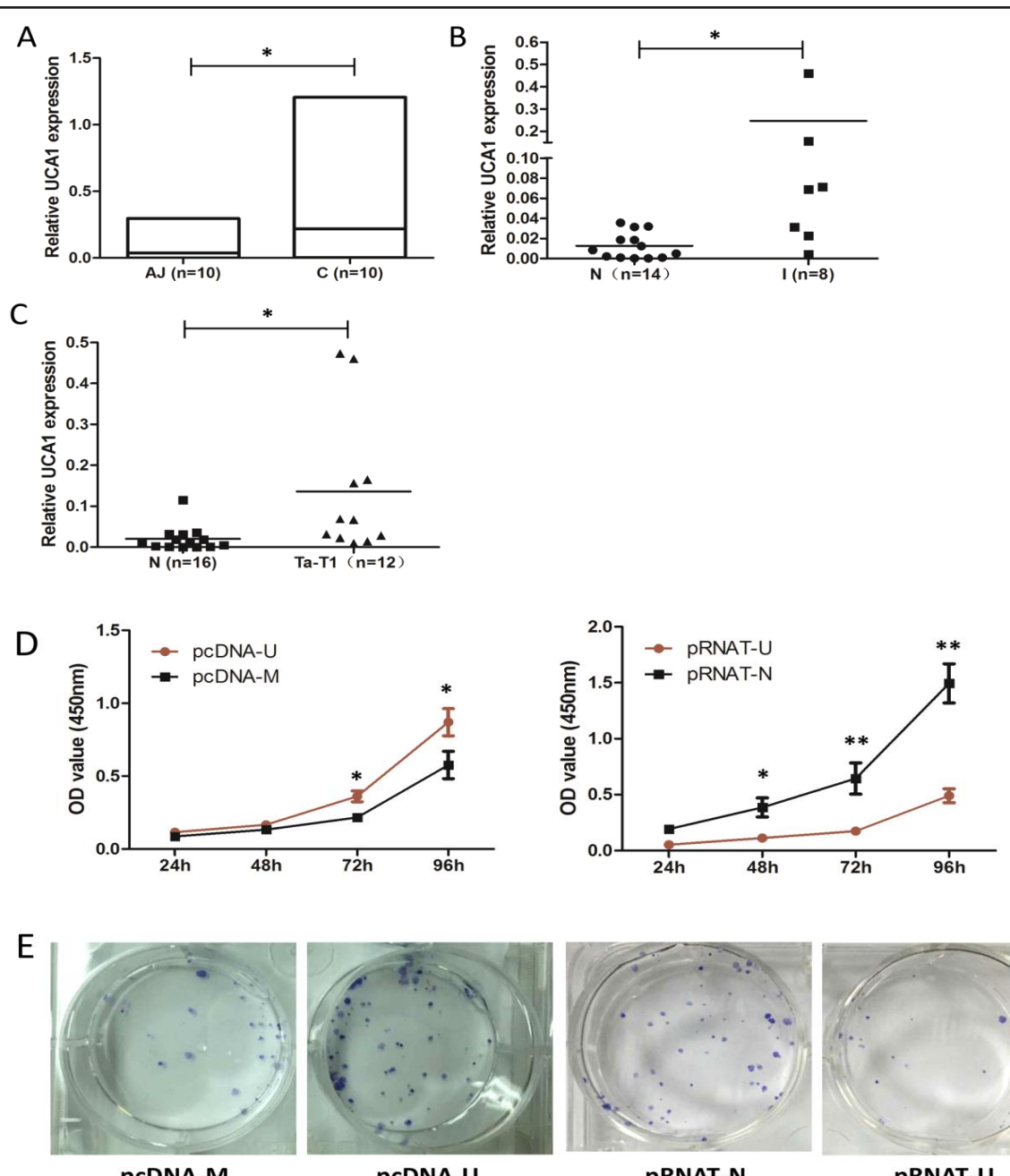

pcDNA-M

pcDNA-U

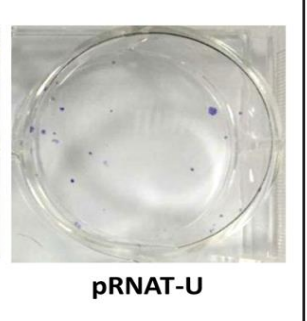

Relative ex-

pression of UCA1 in non-muscle-invasive bladder cancer tissues (Ta-T1) (n=12) and normal bladder tissues $(n=16)$. (D) The CCK8 assay was performed to determine the effects of upregulation and the knockdown of UCA1 expression after transfection for $24,48,72,96 \mathrm{~h}$. (E) The colony-formation assay performed in overexpression and knockdown of UCA1 expression cells after treated for ten days. ${ }^{*} \mathrm{P}<0.05,{ }^{* *} \mathrm{P}<0.01$. Note: pcDNA-M or pcDNA-U: stable cell lines with ectopic expression of UCA1 in UMUC2 cells, constructed with pcDNA3.1(+)/Mock or pcDNA3.1(+)/UCA1; pRNAT-N or pRNAT-U: stable cell lines knockdown of UCA1 in 5637 cells, after transfected with pRNAT-U6.1/Neo-NC or pRNAT-U6.1/Neo-shUCA1, respectively.

\section{UCA1 promotes mitochondrial function in BC cells in vitro}

To investigate whether UCA1 expression may regulate BC mitochondrial function, we detected mitochondrial DNA copy numbers by real-time PCR. Results showed that overexpression of UCA1 (14.4-fold increase) enhanced D-loop expression level in UMUC2, while knockdown of UCA1 (68.1\% decrease) decreased mitochondrial DNA (mtDNA) copy numbers in 5637 (Fig. 2, A and B). To examine whether ATP level is regulated by UCA1, we performed ATP assay, which showed that UCA1 overexpression resulted in increased ATP production (1.4-fold increase). Moreover, ATP levels (28.2\% decrease) were lower after suppression of UCA1 (Fig. 2, C and D). We then analyzed mitochondrial membrane potential by JC-1 fluorescent probe; the results showed that RFP/GFP ratio was significantly increased in UCA1 high-expression cells (1.4-fold increase) compared to control cells. Similarly, the ratio of RFP/GFP (47.7\% decrease) was reduced when we silenced UCA1 in 5637 cells (Fig. 
Fig. 2. UCA1 promotes mitochondrial function of BC cell in vitro. (A) Mitochondrial DNA copy numbers detected by real-time PCR in overexpression of UCA1. (B) Mitochondrial DNA copy numbers in downregulation of UCA1. (C) ATP levels in overexpression of UCA1. (D) ATP levels in downregulation of UCA1. (E) Mitochondrial membrane potential analyzed by JC-1 fluorescent probe (shown as RFP/GFP ratio) in UCA1 high-expression cells. (F) Mitochondrial membrane potential in knockdown of UCA1 in 5637 cells.

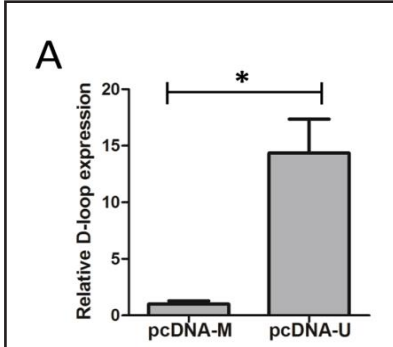

B

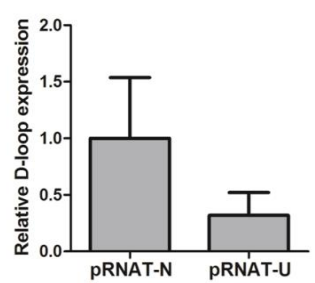

$D$

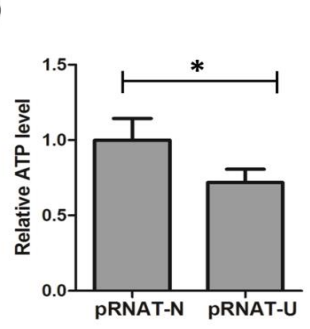

$\mathrm{E}$

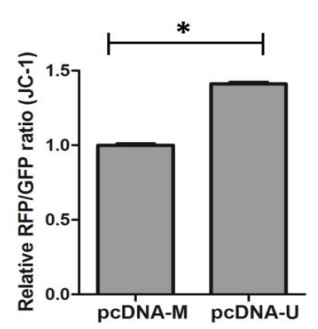

C

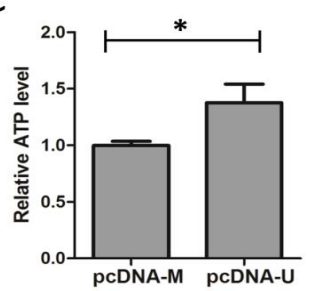

$\mathrm{F}$

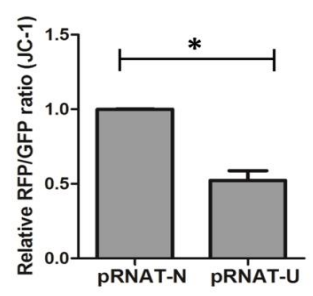

G

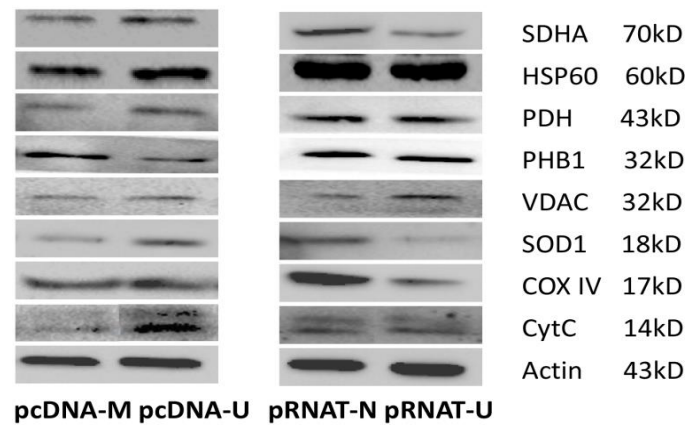

(G) The expression levels of mitochondrial markers (SDHA, HSP60, PDH, PHB1, VDAC, SOD1, COX IV, and CytC) in upregulation and knockdown of UCA1 expression cells by Western blotting. $* \mathrm{P}<0.05$.

2, E and F). In line with this finding, UCA1 enhanced the expression levels of mitochondrial markers (Fig. 2G). These results strongly highlighted the significance of UCA1 expression in enhanced mitochondrial function of BC cells.

\section{UCA1 upregulates ARL2 by inhibiting miR-195-5p}

This study also found that lncRNAs functioned as a ceRNA in mediating the expression of microRNAs and regulating their relevant cellular functions. The RNAHybrid website was used to predict and compare the free energy between two molecules, and the value between UCA1 and miR-195-5p was $-19.6 \mathrm{kcal} / \mathrm{mol}$. In addition, real-time PCR-based microRNA expression was performed to determine the different expression levels of microRNAs among different groups, including miR-195 mimic, NC mimic, pcDNA3.1+ miR-195 mimic, and pcDNA3.1/UCA1+ miR-195 mimic. The results showed that miR-195 expression level $(79 \%$ decrease) was suppressed after ectopic expression of UCA1 (Fig. 3A). Target genes of miR195-5p were predicted by the TargetScan website, and ARL2 was a predicted target gene of miR-195-5p. Moreover, four putative miR-195 binding sites were recognized in the 3'UTR of ARL2 as highly conserved among different species [15]. We next confirmed that UCA1 could enhance ARL2 by inhibiting miR-195-5p ( $81.5 \%$ decrease), determined by real-time PCR and western blotting (Fig. 3, B and C). Furthermore, we detected the relationship among UCA1, miR-195, and ARL2 in 28 bladder tissues, and the results confirmed that RNA expression levels of UCA1 were negatively correlated with those of miR-195 ( $r=-0.544, P<0.01$; Fig. 3D). Meanwhile, the RNA expression levels of ARL2 and miR-195 showed a significant negative 


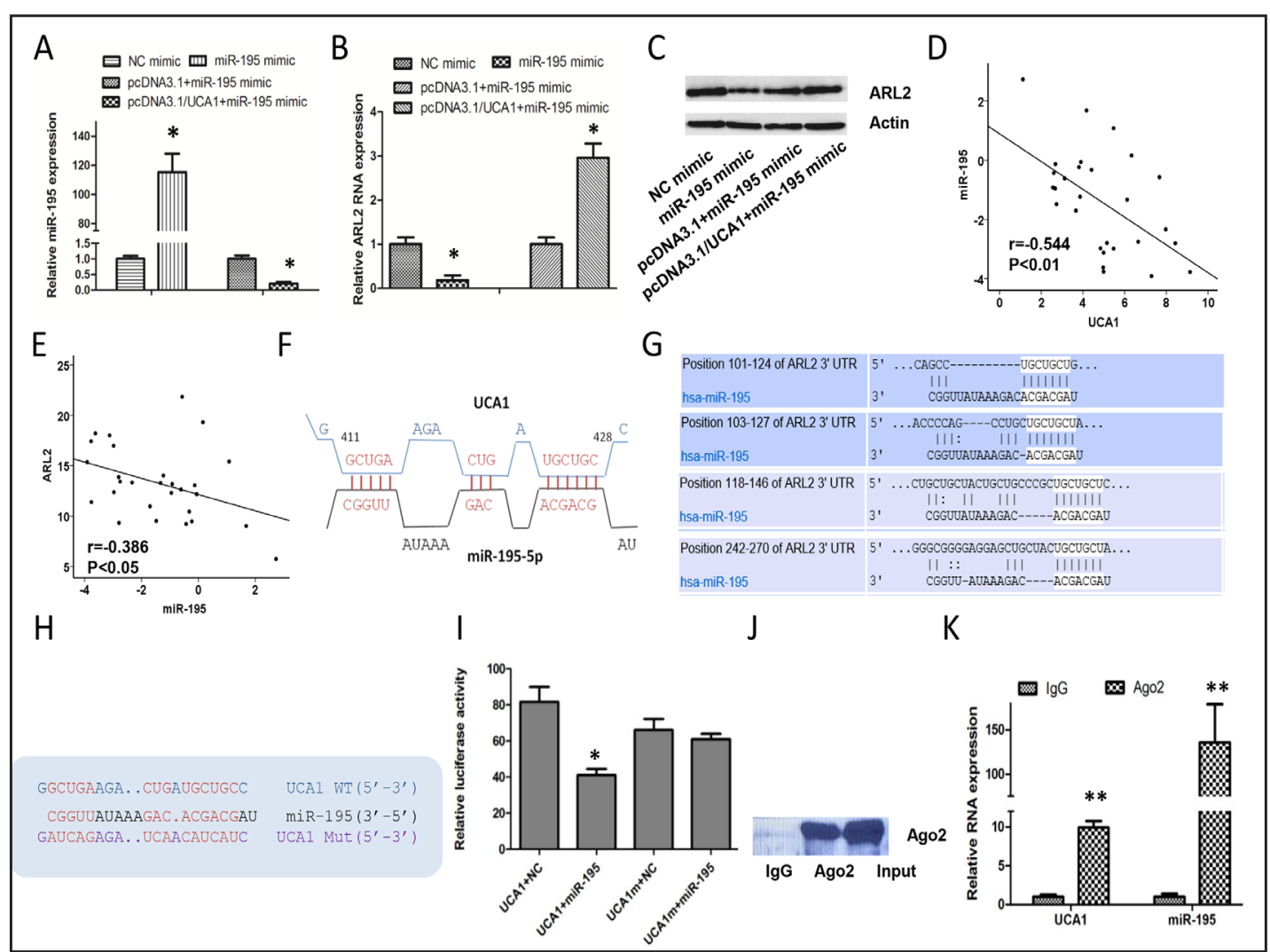

Fig. 3. UCA1 promotes ARL2 expression by inhibiting miR-195-5p. (A) Relative miR-195 expression among different groups, including miR-195 mimic, NC mimic, pcDNA3.1+ miR-195 mimic and pcDNA3.1/UCA1+ miR-195 mimic. (B) Relative expression of the ARL2 mRNA as determined performing qPCR in UMUC2 cells transfected with miR-195 mimic, NC mimic, pcDNA3.1+ miR-195 mimic and pcDNA3.1/UCA1+ miR-195 mimic. (C) Relative expression of the ARL2 protein determined by Western blotting among different groups (miR-195 mimic, NC mimic, pcDNA3.1+ miR-195 mimic and pcDNA3.1/UCA1+ miR-195 mimic). (D) Correlations between UCA1 and miR-195 expression in BC tissues( $r=-0.544, \mathrm{P}<0.01)$. (E) Correlations between miR-195 and ARL2 expression in BC tissues ( $\mathrm{r}=-0.386, \mathrm{P}<0.05)$. (F) A model representing binding sites between UCA1 and miR-195-5p aligned by RNAHybrid software. (G) Schemiatic representation of interaction between miR-195-5p and 3'-UTR of ARL2. (H) A photograph depicted mutation sequence of UCA1 with miR195-5p. (I) Relative luciferase activity was measured 24 hours post transfection treated with pMIR-RepLuc/UCA1-WT or pMIR-Rep-Luc/UCA1-Mut with miR-195-5p mimic. (J) Ago2 protein immunoprecipitated from cell extracts by IgG antibody or Ago2 antibody determined by western blotting. (K) Relative amount of UCA1 and miR-195 bound to IgG of Ago2 tested by Real-time PCR. ${ }^{*} \mathrm{P}<0.05,{ }^{* *} \mathrm{P}<0.01$.

correlation $(r=-0.386, P<0.05$; Fig. 3E). In order to investigate the molecular mechanisms of UCA1 in BC cells, we found that UCA1 was aligned with sequences of miR-195-5p by RNAHybrid software (Fig. 3F). We predicted the interaction between miR-195-5p and 3'UTR of ARL2 according to the TargetScan website (http://www.targetscan.org/mamm_31/) (Fig. 3G). In addition, the prediction was verified through dual-luciferase assay by Zhou et al [15].. This observation verified that miR-195 might negatively regulate ARL2 expression by directly binding to the sequence of ARL2 3'UTR. To further confirm whether UCA1 was a target of miR-195, we first analyzed the UCA1 sequence and the mature chain sequence of miR-195-5p, and the alignment revealed that the "seed region" of miR-195-5p was highly complementary to UCA1. We then designed a mutation sequence of UCA1 with miR-195-5p (Fig. 3H). Furthermore, we utilized dual-luciferase reporter assay to detect the luciferase activity. Our results indicated that miR-195 may reduce the luciferase activity of pMIR-RepLuc/UCA1-WT (49.7\% decrease), while the inhibitory effect was retarded by pMIR-Rep- 


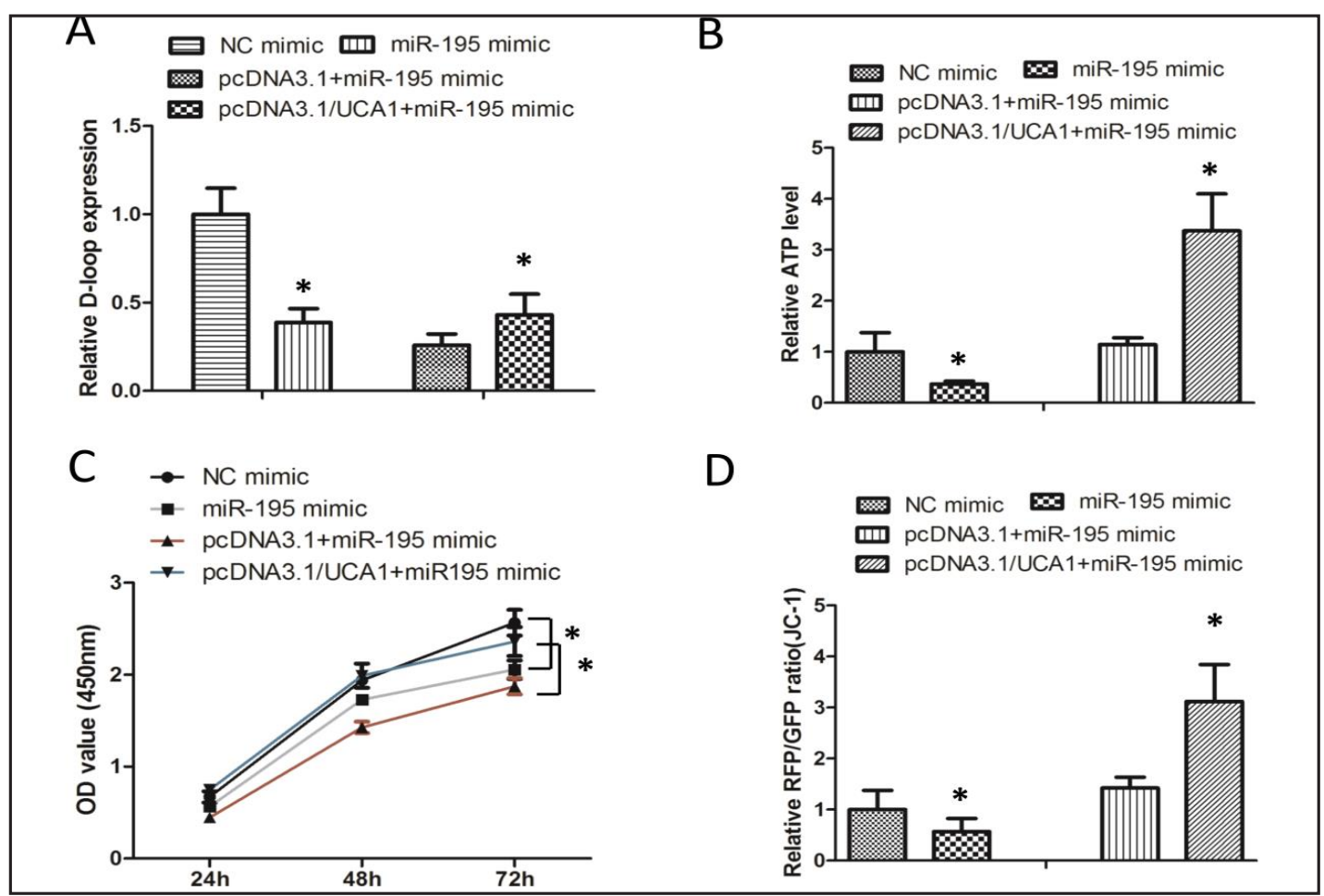

Fig. 4. UCA1 promotes mitochondrial function by depressing miR-195 in BC cells. (A) Relative D-loop expression of UMUC2 cells after transfected with miR-195 mimic, NC mimic, pcDNA3.1+ miR-195 mimic and pcDNA3.1/UCA1+ miR-195 mimic. (B) Relative ATP level in different groups, including miR-195 mimic, NC mimic, pcDNA3.1+ miR-195 mimic and pcDNA3.1/UCA1+ miR-195 mimic. (C) Cellular growth viability of UMUC2 cells after transfected with miR-195 mimic, NC mimic, pcDNA3.1+ miR-195 mimic and pcDNA3.1/ UCA1+ miR-195 mimic. (D) Mitochondrial membrane potential (JC-1) in different groups, including miR195 mimic, NC mimic, pcDNA3.1+ miR-195 mimic and pcDNA3.1/UCA1+ miR-195 mimic). ${ }^{*} \mathrm{P}<0.05$.

Luc/UCA1-Mut (7.7\% decrease) (Fig. 3I). To further confirm whether UCA1 associated with miR-195-5p, RIP experiments were carried out on UMUC2 cells. Ago2 protein was strongly immunoprecipitated from cell extracts by anti-Ago2 antibody (Fig. 3J). RIP assays indicated that UCA1 expression level was higher in Ago2 immunoprecipitates than in the IgG group, and the expression level of miR-195-5p was significantly enhanced in Ago2 complexes compared to that of the control group (Fig. 3K). Taken together, these data indicated that UCA1 promoted ARL2 expression by suppressing miR-195-5p.

\section{UCA1 promotes mitochondrial function by depressing miR-195 in BC cells}

To further clarify whether UCA1 regulated mitochondrial function through suppressing miR-195, we evaluated the mtDNA copy number, ATP production, mitochondrial activity, and cellular growth viability in different groups, including miR-195 mimic, NC mimic, pcDNA3.1+ miR-195 mimic, and pcDNA3.1/UCA1+ miR-195 mimic. These results indicated that miR-195 mimic reduced the expression level of D-loop (61.3\% decrease), and mtDNA copy number elevated after co-transfecting with pcDNA3.1/UCA1 (1.7-fold increase) and miR-195 mimic compared to the control (Fig. 4A). As expected, UCA1 increased mitochondrial ATP level (2.96-fold increase), cell viability, and mitochondrial membrane potential through inhibiting miR-195 (Fig. 4, B-D). These results showed that UCA1 enhanced mitochondrial function by downregulating miR-195.

The effect of ARL2 on mitochondrial function in vitro

To further verify whether ARL2 played an important role in regulating mitochondrial activity, three different ARL2 small interfering RNAs were selected to knock down the ARL2 
Fig. 5. The effect of ARL2 on mitochondrial function in vitro. (A) Relative ARL2 mRNA levels after treated with small interfering RNAs against ARL2 in cell line 5637 was verified by real-time PCR. (B) Relative expression levels of ARL2 protein after treated with si-ARL2 in 5637 cells was determined by western blotting. (C) Relative mtDNA copy number in 5637 cell lines after knockdown of ARL2. (D) Relative ATP production when ARL2 was silenced. (E) Mitochondrial membrane potential analyzed by JC-1 fluorescent probe (shown as RFP/GFP ratio) after knockdown of ARL2. (F) Cellular growth viability after suppression of ARL2 expression in 5637 cells. ${ }^{*} \mathrm{P}<0.05$.

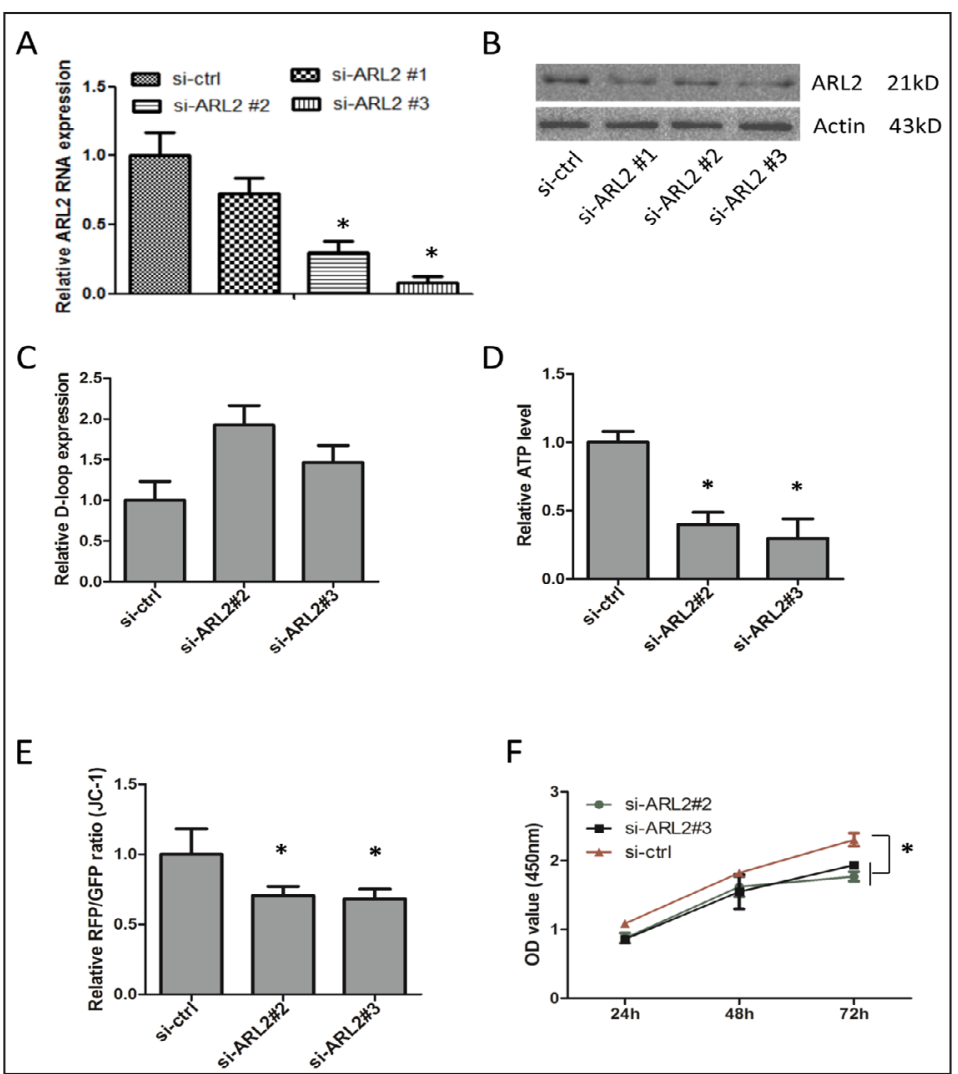

expression in cell line 5637. Successful inhibition of ARL2 expression after transfection with si-ARL2\#2 or si-ARL2\#3 was verified by real-time PCR and western blotting (Fig. 5, A and B). Contrary to our hypothesis, knock down of ARL2 did not decrease mtDNA copy number (1.9-fold and 1.5-fold increases) (Fig. 5C). ATP assay revealed reduced ATP production (60\% and $70 \%$ decreases) in cells when ARL2 was silenced (Fig. 5D). Additionally, mitochondrial membrane potential (JC-1) (29\% and 32\% decreases) and cellular growth viability $(23 \%$ and 16\% decreases) were inhibited after suppression of ARL2 expression (Fig. 5, E-F). These findings revealed that silencing of ARL2 repressed mitochondrial function in BC cells.

UCA1 promotes tumor growth in vivo through the UCA1/miR-195/ARL2 pathway

To determine the effect of UCA1 on tumorigenesis in vivo, pRNAT-N and pRNAT-U cells were subcutaneously injected into nude mice. Knock down of UCA1 significantly inhibited tumor growth in vivo, and presented a significant decrease in tumor volume (26\% decrease) (Fig. 6A). Compared with pRNAT-N, there were significant differences (83\% decrease) in tumor weight after day 20 (Fig. 6B). However, there was no difference in the weight of the mice between two groups (Fig. 6C). Moreover, we analyzed the RNA expression levels of ARL2 and miR-195 by real-time PCR, which showed elevated levels of miR-195 (2.4-fold increase) and decreased RNA levels of ARL2 (68\% decrease) when UCA1 was downregulated (55\% decrease) in vivo (Fig. 6D). As shown in Fig. 6E, HE staining and immunochemistry assay showed that downregulation of UCA1 inhibited tumor growth and decreased protein expression levels of ARL2. Collectively, these data demonstrate that UCA1 plays a pivotal role in mitochondrial metabolism reprogramming in BC by the UCA1/miR-195-5p/ARL2 axis.

\section{Discussion}

Increased understanding of the important role of mitochondria in tumor progression has revealed a promising novel anticancer therapy. Deregulated mitochondrial function in tumor 


\section{Cellular Physiology Cell Physiol Biochem 2017;43:2548-2561

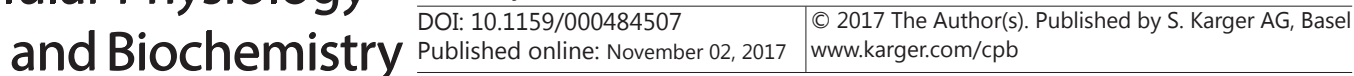

Fig. 6. UCA1 promotes tumor growth in vivo by the UCA1/ miR-195/ARL2 axis. (A-C) Tumor size, tumor volume, and mouse weight of subcutaneous cancer model of pRNAT$\mathrm{U}$ and pRNAT-N cells are representation. (D) Relative RNA expression of UCA1, miR-195 and ARL2 in tumor tissues. (E) HE staining and protein expression level of ARL2 detected by immunochemistry staining $(400 \times)$. * $\mathrm{P}<0.05$.

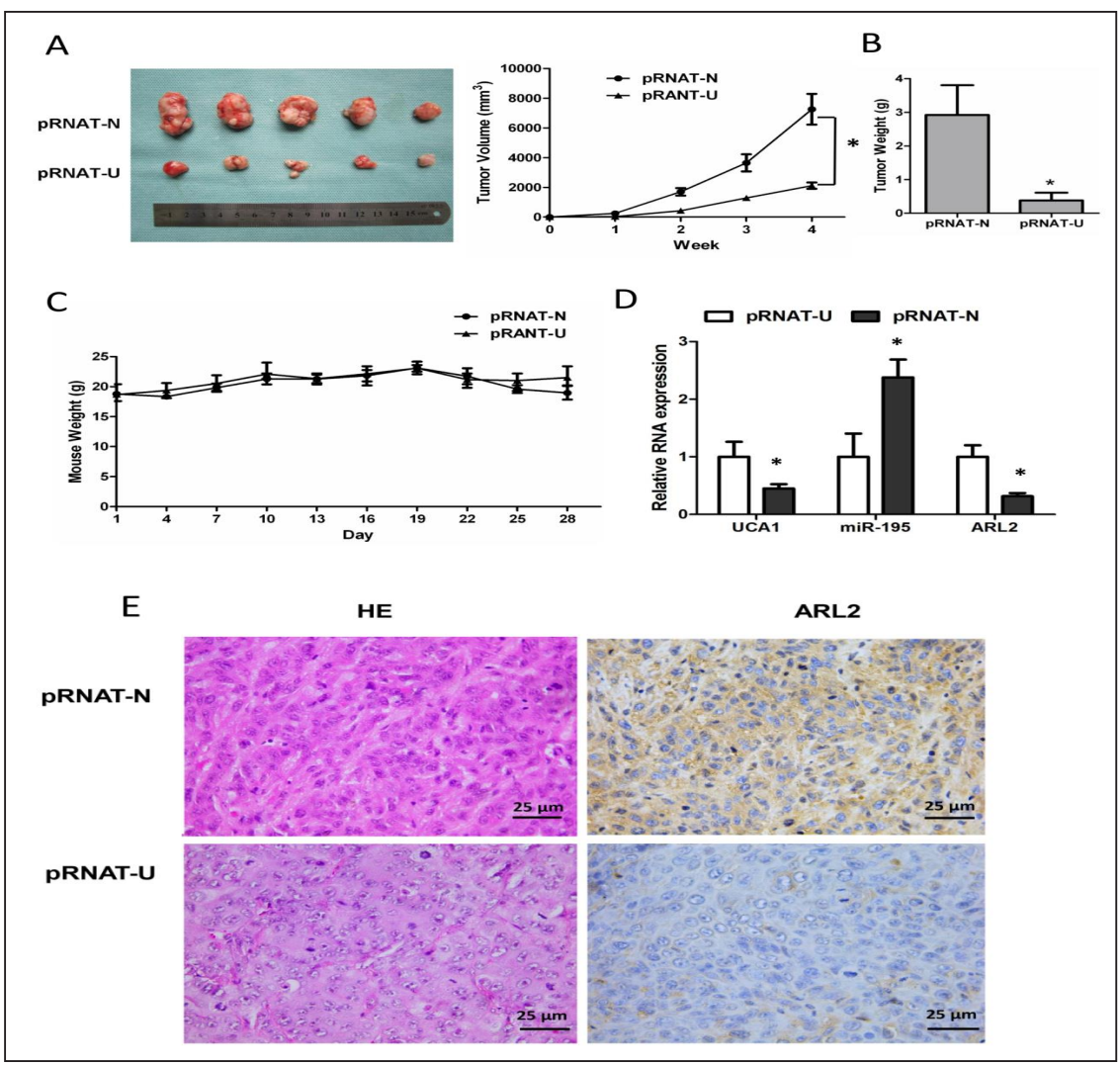

cells, as originally proposed by Otto Warburg, inhibited oxidative phosphorylation [17]. However, mitochondria are not simply innocent bystanders in tumor development, because some tumor cells are more dependent on mitochondrial metabolism. UCA1 is involved in regulation of the Warburg effect through the mTOR/STAT3/miR-143/HK2 signaling pathway, which strongly suggests that UCA1 participates in metabolic reprogramming. However, the mechanism whereby UCA1 participates in tumor mitochondrial function has not been clarified.

Recently, it has been reported that IncRNAs may act as ceRNAs to regulate gene expression, playing important roles in tumor progression [18]. It has been recently demonstrated that IncRNA UCA1 promotes BC cell migration and invasion by modulating FSCN1 as a ceRNA for miR-145. Moreover, UCA1 functions as a ceRNA of miR-16 to contribute to imatinib resistance in chronic myeloid leukemia cells [19]. In addition, UCA1 promotes cellular proliferation by acting as a ceRNA against Sox4 in esophageal cancer [20]. Therefore, we hypothesized that UCA1 can also acts as a decoy miRNA in regulation of mitochondrial function in BC. Previous studies showed that UCA1 was a negative prognostic factor for BC [21], and our clinical data demonstrated similar results, showing that UCA1 expression in bladder tumor tissues was significantly higher than that of adjacent bladder tissues. Specifically, UCA1 expression was remarkably higher in phase I and Ta-T1 than in normal bladder tissues. Our study also reported a positive correlation between UCA1 expression levels and malignant tumor cells. In addition, UCA1 remarkably increased cell viability and clone formation in UMUC2 cells, while knockdown of UCA1 effectively reversed this. In order to test whether UCA1 was involved in the regulation of mitochondrial function, we then detected mitochondrial biogenesis by assessing the mtDNA copy number, ATP production, mitochondrial membrane potential, and mitochondrial-associated protein expression levels. The results suggested that UCA1 may act as an oncogene and could play an important role in mitochondrial metabolism in bladder tumors.

Although it has been verified that UCA1 may modulate mitochondrial metabolism in bladder cancer, the underlying mechanism remains unclear. microRNAs play important 
regulatory roles in bladder cancer development and progression via manipulating cell growth, proliferation, migration and invasion $[22,23]$.To detect whether UCA1 may act as ceRNAs in the regulation of bladder tumors, we predicted miRNAs binding to UCA1 by RNAHybrid software and determined them by real-time PCR. Importantly, we confirmed that levels of miR-195 were downregulated by UCA1 expression in BC cell lines. Previous studies have shown that miR-195 acts as a tumor suppressor in multiple types of cancer and has functions in various pathways [24-26].

In this study, we screened candidate target genes of miR-195 by bioinformatics analysis, to determine which may be involved in modulating mitochondrial function. Bioinformatics analysis revealed that GLUD1 and ARL2 were ideal targets of miR-195. mRNA and protein levels of GLUD1 and ARL2 were further detected, and the results showed that GLUD1 expression levels were not abolished by treatment with miR-195 mimics (unpublished data). On the other hand, miR-195 expression was negatively correlated with ARL2 expression. Moreover, miR-195 directly suppressed ARL2 mRNA and protein levels in bladder tumor cells, indicating that miR-195 may be a tumor suppressor gene that decreases ARL2 expression in BC. Additionally, it has been reported that ARL2 expression was directly regulated by miR-195 in human neural progenitor cells [20]. These findings suggest that ARL2 is in turn negatively regulated by miR-195.

In order to investigate whether UCA1 increased mitochondrial function by decoying miR-195 in bladder tumor cells, we confirmed the mtDNA copy number, ATP production, cell viability, and mitochondrial activity in different groups. As expected, miR-195 decreased the mtDNA copy number, ATP production, cell viability, and mitochondrial activity. Meanwhile, UCA1 enhanced mitochondrial efficacy by sequestering miR-195 in bladder tumor cells. We also treated bladder tumor cells with ARL2-siRNA to investigate whether the suppressed mitochondrial function was affected by ARL2 expression. The results showed that knockdown of ARL2 inhibited mitochondrial function in BC cells. In addition, UCA1 could promote bladder tumor progression and ARL2 expression in vivo (Fig. 6). Altogether, UCA1 enhanced mitochondrial function, cell viability, and mitochondrial membrane potential through the UCA1-miR-195-ARL2 signaling pathway in BC cells.

In conclusion, we have demonstrated that UCA1 is involved in mitochondrial metabolism and mitochondrial function in bladder tumors. It has been shown that UCA1 acts as a ceRNA to inhibit the expression level of miR-195, resulting in elevated ARL2 expression, which is essential for mitochondrial function. Here, for the first time, we have highlighted that UCA1 promotes mitochondrial function and achieves this through the UCA1-miR-195-ARL2 signaling network.

\section{Acknowledgments}

This work was supported by National Natural Science Foundation of China (No. 81572735 and 81772735).

\section{Disclosure Statement}

None.

\section{References}

$>1$ Chavan S, Bray F, Lortet-Tieulent J, Goodman M, Jemal A: International variations in bladder cancer incidence and mortality. Eur Urol 2014;66:59-73.

-2 Hirschey MD, DeBerardinis RJ, Diehl AM, Drew JE, Frezza C, Green MF, Jones LW, Ko YH, Le A, Lea MA, Locasale JW, Longo VD, Lyssiotis CA, McDonnell E, Mehrmohamadi M, Michelotti G, Muralidhar V, Murphy MP, Pedersen PL, Poore B, Raffaghello L, Rathmell JC, Sivanand S, Vander Heiden MG, Wellen KE; Target Validation Team: Dysregulated metabolism contributes to oncogenesis. Semin Cancer Biol 2015;35:S129150. 


\section{Cellular Physiology Cell Physiol Biochem 2017;43:2548-2561 \begin{tabular}{l|l|l|} 
DOI: 10.1159/000484507 & O 2017 The Author(s). Published by S. Karger AG, Basel \\
wwww.karger.com/cpb
\end{tabular} and Biochemistry $\frac{\text { Published online: November 02, 2017 }}{\text { Li et al.: UCA1/MiR-195/ARL2 in Mitochondria of BC }}$}

3 Ru P, Williams TM, Chakravarti A, Guo D: Tumor metabolism of malignant gliomas. Cancers (Basel) 2013;5:1469-1484.

-4 Warburg 0: On respiratory impairment in cancer cells. Science 1956;124:269-270.

5 Roadmap Epigenomics Consortium, Kundaje A, Meuleman W, Ernst J, Bilenky M, Yen A, Heravi-Moussavi A, Kheradpour P, Zhang Z, Wang J: Integrative analysis of 111 reference human epigenomes. Nature 2015;518:317-330.

6 Guttman M, Rinn JL: Modular regulatory principles of large noncoding RNAs. Nature 2012;482:339-346.

7 Batista PJ, Chang HY: Long noncoding RNAs: cellular address codes in development and disease. Cell 2013;152:1298-1307.

8 Shi X, Sun M, Liu H, Yao Y, Song Y: Long non-coding RNAs: a new frontier in the study of human diseases. Cancer Lett 2013;339:159-166.

-9 Yang C, Li X, Wang Y, Zhao L, Chen W: Long non-coding RNA UCA1 regulated cell cycle distribution via CREB through P13-K dependent pathway in bladder carcinoma cells. Gene 2012;496:8-16.

10 Xue M, Pang H, Li X, Li H, Chen W: Long non-coding RNA urothelial cancer-associated 1 promotes bladder cancer cell migration and invasion by way of the hsa-miR-145-ZEB1/2-FSCN1 pathway. Cancer Sci 2016;107:18-27.

11 Li Z, Li X, Wu S, Xue M, Chen W: Long non-coding RNA UCA1 promotes glycolysis by upregulating hexokinase 2 through the mTOR-STAT3/microRNA143 pathway. Cancer Sci 2014;105:951-955.

12 Clark J, Moore L, Krasinskas A, Way J, Battey J, Tamkun J, Kahn RA: Selective amplification of additional members of the ADP-ribosylation factor (ARF) family: cloning of additional human and Drosophila ARF-like genes. Proc Natl Acad Sci USA 1993;90:8952-8956.

13 Newman LE, Schiavon CR, Zhou C, Kahn RA: The abundance of the ARL2 GTPase and its GAP, ELMOD2, at mitochondria are modulated by the fusogenic activity of mitofusins and stressors. PLoS One 2017;12:e0175164.

14 Nishi H, Ono K, Iwanaga Y, Horie T, Nagao K, Takemura G, Kinoshita M, Kuwabara Y, Mori RT, Hasegawa K, Kita T, Kimura T: MicroRNA-15b modulates cellular ATP levels and degenerates mitochondria via Arl2 in neonatal rat cardiac myocytes. J Biol Chem 2010;285:4920-4930.

15 Zhou Y, Jiang H, Gu J, Tang Y, Shen N, Jin Y: MicroRNA-195 targets ADP-ribosylation factor-like protein 2 to induce apoptosis in human embryonic stem cell-derived neural progenitor cells. Cell Death Dis 2013;4:e695

16 Li HJ, Li X, Pang H, Pan JJ, Xie XJ, Chen W: Long non-coding RNA UCA1 promotes glutamine metabolism by targeting miR-16 in human bladder cancer. Jpn J Clin Oncol 2015;45:1055-1063.

17 Vander Heiden MG, Cantley LC, Thompson CB: Understanding the Warburg effect: the metabolic requirements of cell proliferation. Science 2009;324:1029-1033

18 Tay Y, Rinn J, Pandolfi PP: The multilayered complexity of ceRNA crosstalk and competition. Nature 2014;505:344-352.

19 Xiao Y, Jiao C, Lin Y, Chen M, Zhang J, Wang J, Zhang Z: IncRNA UCA1 Contributes to Imatinib Resistance by Acting as a ceRNA Against miR-16 in Chronic Myeloid Leukemia Cells. DNA Cell Biol 2017;36:18-25.

-20 Jiao C, Song Z, Chen J, Zhong J, Cai W, Tian S, Chen S, Yi Y, Xiao Y: IncRNA-UCA1 enhances cell proliferation through functioning as a ceRNA of Sox4 in esophageal cancer. Oncol Rep 2016;36:2960-2966.

-21 Wang XS, Zhang Z, Wang HC, Cai JL, Xu QW, Li MQ, Chen YC, Qian XP, Lu TJ, Yu LZ, Zhang Y, Xin DQ Na YQ Chen WF: Rapid identification of UCA1 as a very sensitive and specific unique marker for human bladder carcinoma. Clinical Cancer Research 2006;12:4851-4858.

22 Zhi Y, Pan J, Shen W, He P, Zheng J, Zhou X, Lu G, Chen Z, Zhou Z: Ginkgolide B inhibits human bladder cancer cell migration and invasion through microRNA-223-3p. Cell Physiol Biochem 2016;39:1787-1794

23 Sun DK, Wang JM, Zhang P, Wang YQ: MicroRNA-138 regulates metastatic potential of bladder cancer through zeb2 Cell Physiol Biochem 2015;37:2366-2374.

24 Yongchun Z, Linwei T, Xicai W, Lianhua Y, Guangqiang Z, Ming Y, Guanjian L, Yujie L, Yunchao H: MicroRNA-195 inhibits non-small cell lung cancer cell proliferation, migration and invasion by targeting MYB. Cancer Lett 2014;347:65-74.

25 Luo Q, Wei C, Li X, Li J, Chen L, Huang Y, Song H, Li D, Fang L: MicroRNA-195-5p is a potential diagnostic and therapeutic target for breast cancer. Oncol Rep 2014;31:1096-1102.

26 Wang X, Wang J, Ma H, Zhang J, Zhou X: Downregulation of miR-195 correlates with lymph node metastasis and poor prognosis in colorectal cancer. Med Oncol 2012;29:919-927. 